KIDNEY CANCER

\title{
Activation of oncogenes driven by VHL loss in ccRCC
}

4

Knockdown

of ZNF395

inhibited

colony

formation,

decreased

cell viability, and increased

apoptosis
The most comprehensive profiling of histone marks in clear cell renal cell carcinoma (ccRCC) undertaken to date has revealed that von HippelLindau (VHL) loss contributes considerably to enhancer remodelling. Furthermore, a novel master regulator of ccRCC pathogenesis ZNF395 - has been identified. These results provide mechanistic insights into ccRCC development and progression and provide new potential treatment targets.

Histone profiling of three marks in ccRCC tumours, patient-matched, tumour-derived cell lines, and nonmalignant kidney cell lines showed that the cis regulatory landscape of ccRCC is aberrant, having extensive genome-wide alterations. Both gain and loss of promoters and enhancers occurred, and gained promoters and enhancers had increased chromatin accessibility and long noncoding RNA expression and decreased DNA methylation.

Genes modified by tumourspecific regulatory elements were identified using three methods to assign enhancers. These analyses showed that gained enhancers have a ccRCC-specific signature, enriched in disease-specific features such as the HIF1a regulatory network and cell metabolism, whereas gained promoters were enriched at features involved in general cancerpromoting processes, such as the cell cycle and transcription.

Examination of the regulatory landscape of super-enhancers showed that 1,157 were gained and 294 were lost in ccRCC tumours. Among the putative targets of these superenhancers were well-known oncogenes, but several lesser-known genes were also identified, which were overexpressed in tumours compared with nonmalignant samples and were unique to ccRCC.

ZNF395 was overexpressed in ccRCC tumours and kidney cancer cell lines. Knockdown of ZNF395 inhibited colony formation, decreased cell viability, and increased apoptosis, but did not affect nonmalignant kidney cells. The ZNF395 super-enhancer was active in ccRCC cells, but not in nonmalignant cells, and it was exclusively overexpressed in ccRCC in the cancers profiled in TCGA. In vivo, ablation of ZNF395 inhibited xenograft tumour growth.

Restoration of VHL in RCC cell lines delayed tumour growth in vivo and caused more pronounced changes to enhancers and superenhancers than to promoters. Only gained enhancers with $\mathrm{H} 3 \mathrm{~K} 27 \mathrm{ac}$ depletion were active in $\mathrm{VHL}$ mutated ccRCC cell lines, and only these enhancers were associated with downregulation of their putative target genes on VHL restoration. Further analysis suggested that $V H L$ loss contributes to enhancer malfunction in ccRCC and that $V H L$ restoration can result in loss of enhancer identity caused by codepletion of histone marks.

HIF $2 \alpha$ was the most enriched motif at $V H L$-responsive enhancers with $\mathrm{H} 3 \mathrm{~K} 27 \mathrm{ac}$ depletion, and its expression was downregulated on $V H L$ restoration. Gained enhancers had HIF2 $\alpha$ occupancy twice that of tumour-specific promoters. HIF1 $\alpha$ was not enriched at enhancers with $\mathrm{H} 3 \mathrm{~K} 27 \mathrm{ac}$ depletion and localized to promoter-proximal regions, whereas
HIF2 $\alpha$ localized to distal regions. More overlap of HIF2 $a$ binding sites with gained enhancers was observed than for HIF1 a binding sites; the opposite was found for gained promoters. Knockdown of HIF2A did not recapitulate $V H L$ restoration in terms of gene expression and gained enhancers; however, correlation between the two conditions was increased for genes close to HIF $2 \alpha$ and HIF $2 \alpha$-bound enhancers and super-enhancers.

Profiling of histone acetyltransferase p300 showed enrichment at gained enhancers compared with lost enhancers in RCC cell lines. Restoration of VHL in VHL-depleted cells decreased p300 binding; however, promoter-enhancer interactions were preserved. HIF2A knockdown also decreased p300 recruitment.

These results show that the cis regulatory landscape is abberant in RCC and identify the VHL-dependent enhancer that is required for ZNF395 expression, which has a functional role in ccRCC tumorigenesis. These data provide new potential treatment targets for this disease.

Louise Stone

ORIGINAL ARTICLE Yao, X. et al. VHL deficiency drives enhancer activation of oncogenes in clear cell renal cell carcinoma. Cancer Discov. http:// dx.doi.org/10.1158/2159-8290.CD-17-0375 (2017)

O

